\title{
Combination Therapy of Antiepileptic Drugs (AEDS) With Safe Natural Anticonvulsant Agent

\author{
${ }^{1}$ Department of Basic Sciences, Faculty of Veterinary Medicine, Amol University of Special Modern Technologies, Amol-Iran
} \\ Hakimeh Gavzandarounkola ${ }^{1^{*}}$ and Mohammad Sayyah ${ }^{2}$ \\ ${ }^{2}$ Department of Physiology and Pharmacology, Pasteur Institute of Iran, Tehran, Iran
}

*Corresponding author: Hakimeh Gavzandarounkola, Department of Basic Sciences, Faculty of Veterinary Medicine, Amol University of Special Modern Technologies, Amol-Iran, Tel: +98 21 66953311-20; E-mail: h.gavzan@hotmail.com

Rec date: Nov 07, 2016; Acc date: Dec 09, 2016; Pub date: Dec 18, 2016

Copyright: () 2016 Gavzandarounkola H, et al. This is an open-access article distributed under the terms of the Creative Commons Attribution License, which permits unrestricted use, distribution, and reproduction in any medium, provided the original author and source are credited.

\section{Commentary}

Epilepsy is the third most common neurological disorder after stroke and Alzheimer's disease. Treatment of epilepsy has advanced during the past three decades by several third generation antiepileptic drugs (AEDs). Yet, resistance to AEDs, as well as intolerability in 20\% to $30 \%$ of the patients, generates demands for developing new drugs or strategies for treatment of epilepsy [1]. Furthermore, AEDs side effects and their toxicities are the other problem of drug treatment. Combination therapy is one of the most recommended strategies for treatment of refractory epilepsy. It is based on synergistic anticonvulsant action, an antagonistic action with respect to adverse effects, or both. Moreover, combination therapy could minimize side effects and toxicities of conventional AEDs by lowering of drug doses. Combination therapy of conventional AEDs with newer anticonvulsant agents could be useful because new mechanisms of synergism may be recognized.

There are several types of combination therapy in preclinical test. Isobolografic analysis and COMPUSYN software are two common methods. Isobolografic analysis compares the dose of each agent that produced anticonvulsant effect in 50\% of animals (ED50) alone and in co-administration by log-probit analysis. In this method, animals tested by several fixed-ratio combinations e.g. 1:1, 1:3 and 3:1 were calculated from ED50 of each drug used alone. These fixed-ratio combinations are equivalent to additive doses theoretically calculated. Then these fixed-ratio combinations tested in animals and determined the experimental ED50 of fixed-ratio combinations. Significant lowering of experimental ED50 of fixed-ratio combinations than theoretical ED50 of them show synergistic effect. [2-4]. In COMPUSYN software method, degree of drug interaction was determined according to combination index (CI) method. CI is obtained from the following equation: $\mathrm{CI}=1(\mathrm{Dx}) 1+2(\mathrm{Dx}) 2$ where $\mathrm{D} 1$ and D2 are the doses of two drugs used in mixture. (D) $1+$ (D)2 in combination' produces $\mathrm{x} \%$ protection. $(\mathrm{Dx}) 1$ and $(\mathrm{Dx}) 2$ are $\mathrm{D} 1$ and $\mathrm{D} 2$ alone that shows $\mathrm{x} \%$ protection [5]. A CI value equal to 1 indicates additive interaction. A CI smaller than 1 denotes synergism, and a CI greater than 1 suggests antagonism. An isobologram plot was drawn by COMPUSYN software. The isobologram is a graph of equipotency doses for two drug combinations, which is simply created by setting the CI equation equal to 1 for EDx [6]. Co-administration strategy of the safe and inexpensive natural anticonvulsant compounds with AEDs could be favourably regarded in clinical studies of epilepsy treatment.

Omega-3 polyunsaturated fatty acids ( $n-3$ PUFAs) as one of the anticonvulsant natural agent have been proposed in recent years to treat epilepsy [7]. They are dietary lipids, which constitute $30 \%$ of brain lipids. Among the n-3 PUFAs, only DHA is the most abundant and the most bioactive fatty acid in the brain [7]. Numerous in vitro, in vivo, and clinical studies have evaluated possible anticonvulsant activity of DHA, with positive or negative results. According to the nonclinical studies, anticonvulsant potency of DHA is much lower than that of the current standard AEDs [7-14]. Interestingly, by Isobolografic analysis, our last study showed that DHA at ED25 caused a 3.6-fold increase in potency of valproate as its ED50 value on co-administration DHA and VPA against seizures induced by PTZ. Moreover, a 4.9-fold increase in potency of lamotrigine occurred, as its ED50 value against generalized kindled seizures induced by rapid amygdala kindling. COMPUSYN analysis confirmed a synergistic interaction in anticonvulsant activity between DHA and both AEDs: valproate and lamotrigine [13]. This finding shows the need of further preclinical and clinical studies on safe natural anticonvulsant agents like DHA as a component of combination therapy of epilepsy. Furthermore, in recent years there are some reports regarding the central effects of effective natural agent. So, it seems to be useful that the combination therapy of effective natural agent with conventional drugs be investigated on another neurological disorders.

In addition, some studies suggest that combining drugs with different mechanisms of action is more effective against seizure than drugs with similar or overlapping pharmacological properties [15]. So, clearing of anticonvulsant mechanisms of effective safe natural agents like DHA could be useful to select the sufficient complementary drugs on combination therapy with increasing the efficacy and tolerability of drugs as a result.

Evidence-Based Medicine (EBM) provides the classified information on the basis of published data for approaching the best clinical treatment decision making [16]. The optimal clinical application of co-administration of AEDs with natural anticonvulsant agents needs to the systematic categories of studies regarding evidencebased medicine (EBM). In this case as well as clinical mono or polytherapy by AEDs [17-21], EBM could constitute aspects such as:

1. The anticonvulsant action mechanism of AEDs and natural anticonvulsant agents.

2. Efficacy, tolerability and side effect of AEDs and natural anticonvulsant agents in monotherapy and co-administration respected to the kind of seizure, age, condition and neurological and cognitive impairment with epilepsy of patient.

3. The pharmacokinetic and pharmacodynamics interaction between AEDs and natural anticonvulsant agents in co-administration. 
Citation: Gavzandarounkola H, Sayyah M (2016) Combination Therapy of Antiepileptic Drugs (AEDS) With Safe Natural Anticonvulsant Agent. J Neurol Disord 4: 323. doi:10.4172/2329-6895.1000323

Page 2 of 2

\section{References}

1. Löscher W, Klitgaard H, Twyman RE, Schmidt D (2013) New avenues for anti-epileptic drug discovery and development. Nat Rev Drug Discov 12: 757-776.

2. Litchfield JT, Wilcoxon F (1949) A simplified method of evaluating doseeffect experiments. J Pharmacol Exp Ther 96: 99-113.

3. Loewe S (1953) The problem of synergism and antagonism of combined drugs. Arzneimittelforschung 3: 285-290.

4. Tallarida RJ (2000) Drug synergism and dose-effect data analysis. Chapman \& Hall/CRC, Boca Raton.

5. Chou TC (2006) Theoretical basis, experimental design, and computerized simulation of synergism and antagonism in drug combination studies. Pharmacol Rev 58: 621-681.

6. Chou TC (2010) Drug combination studies and their synergy quantification using the Chou-Talalay method. Cancer Res 70: 440-446.

7. Taha AY, Burnham WM, Auvin S (2010a) Polyunsaturated fatty acid and epilepsy. Epilepsia 51: 1348-1358.

8. Taha AY, Jeffrey MA, Tasha NM, Bala S, Burnham WM (2010b) Acute administration of docosahexaenoic acid increase resistance to pentylenetetrazole-induced seizure in rats. Epilepsy Behav 17: 336-343.

9. Musto AE, Gjorstrup P, Bazan NG (2011) The omega-3 fatty acid-derived neuroprotectin D 1 limits hippocampal hyperexcitability and seizure susceptibility in kindling epileptogenesis. Epilepsia 52: 1601-1608.

10. Taha AY, Trepanier MO, Ciobanu FA, Taha NM, Ahmed M, et al. (2013) A minimum of 3 months of dietary fish oil supplementation is required to raise amygdaloid afterdischarge seizure thresholds in rats, implications for treating complex partial seizures. Epilepsy Behav 27: 49-58.

11. Trépanier MO, Lim J, Lai TK, Cho HJ, Domenichiello AF, et al. (2014) Intraperitoneal administration of docosahexaenoic acid for 14 days increases serum unesterified DHA and seizure latency in the maximal pentylenetetrazole model. Epilepsy Behav 33: 138-143.

12. Trépanier MO, Taha AY, Mantha RL, Ciobanu FA, Zeng QH, et al. (2012) Increases in seizure latencies induced by subcutaneous docosahexaenoic acid are lost at higher doses. Epilepsy Res 99: 225-232.

13. Gavzan H, Sayyah M, Sardari S, Bbapour V (2015) Synergistic effect of docosahexaenoic acid on anticonvulsant activity of valproic acid and lamotrigine in animal seizure models. Naunyn-Schmiedeberg's Arch Pharmacol 388(10): 1029-1038.

14. Willis S, Samala R, Rosenberger TA, Borges K (2009) Eicosapentaenoic and docosahexaenoic acids are not anticonvulsant or neuroprotective in acute mouse seizure models. Epilepsia 50: 138-142.

15. Brodie MJ, Sills GJ (2011) Combining antiepileptic drugs-Rational polytherapy? Seizure 20: 369-375.

16. Evidence-Based Medicine Working Group (1992) Evidence-based medicine. A new approach to teaching the practice of medicine. JAMA 268: 2420-2425.

17. Wiebe S (2013) Definition of drug-resistant epilepsy: Is it evidence based? Epilepsia 54: 9-12.

18. Guerreiro CAM (2008) Guidelines for drug treatment of epilepsy. Arq Neuropsiquiatr 66: 591-599.

19. Bielen I (2005) Dilemmas of contemporary epilepsy pharmacotherapy. Acta Med Croatica 59: 1-6.

20. Armijo JA, Sanchez B, González AB (2002) Evidence based treatment of epilepsy. Rev Neurol 1: 59-73.

21. Deckers CLP, Czuczwar SJ, Hekster YA, Keyser A, Kubova H, et al. (2000) Selection of antiepileptic drug polytherapy based on mechanisms of action: The evidence reviewed. Epilepsia 41: 1364-1374. 